\title{
Análisis proximal de la harina de biofloc del cultivo de tilapia roja (Oreochromis sp.)
}

\section{Proximal analysis of biofloc flour of red tilapia (Oreochromis sp.) farming}

\author{
Dr. Jorge Rodríguez Tobar ${ }^{1}$ \\ jrodriguez@uteq.edu.ec \\ Paola Suin Miranda ${ }^{1}$ \\ psuin@uteq.edu.ec \\ Dr. Martin González Veliz ${ }^{1}$ \\ mgonzalez@uteq.edu.ec \\ Ing. Roque Vivas Moreira ${ }^{1}$ \\ rviva@uteq.edu.ec \\ Dr. Raúl Díaz Ocampo ${ }^{1}$ \\ rdiaz@uteq.edu.ec \\ Dra. Yenny Torres Navarrete 1 \\ ytorres@uteq.edu.ec \\ Ing. Rossy Rodríguez Castro ${ }^{1}$ \\ rrodriguez@uteq.edu.ec
}

Recibido: 1/07/2018, Aceptado: 1/09/2018

\begin{abstract}
RESUMEN
La harina de biofloc analizada resultó de la recolección y deshidratación de los sólidos suspendidos en el agua con tecnología biofloc de crianza de tilapias, se utilizó un módulo con 15 tanques de 200 litros cada uno, los tanques fueron inoculados con biofloc procedente de un estanque, para la aireación y movimiento del agua se utilizó un blower, el aire fue conducido mediante una manguera de 0.5 " con una válvula que regulaba el paso del aire y difundido en el agua a través de una manguera, se utilizaron 150 tilapias machos con peso promedio de 200 gramos. Los peces fueron distribuidos aleatoriamente en los 15 tanques, se utilizó cinco repeticiones para cada tratamiento. Los peces de los tratamientos fueron alimentados con raciones de 24, 28 y $32 \%$ de proteína y tasa de alimentación de 4 . Los parámetros ambientales y del agua fueron temperatura promedio del agua 24.5, pH6.8, alcalinidad 120 ppm, amonio no ionizado 0.04 , solidos sedimentables $50 \mathrm{ml} / \mathrm{l}$. Para la obtención del residuo biofloc se utilizó un decantador de separación de la parte sólida del líquido, el residuo obtenido fue deshidratado y analizado. El objetivo fue conocer las propiedades nutricionales y organolépticas de la harina biofloc, en dependencia del alimento consumido por los peces. Los mayores promedios encontrados fueron: proteína $30,65 \pm 0.38$, grasa $1.66 \pm 0.35$, materia seca $89.84 \pm 0.93$, ceniza $23.34 \pm 7.68$, fibra $17.76 \pm 7.68$; el olor fue agradable, presentó color marrón y el $50 \%$ de la harina estaba constituido por partículas con diámetro de $170 \mu \mathrm{m}$.
\end{abstract}

${ }^{1}$ Docentes de la Universidad Técnica Estatal de Quevedo. Ecuador 
Palabras clave: materia orgánica, biofloc, cultivo de tilapia, tecnology

\section{ABSTRACT}

The biofloc meal analyzed was the result of the collection and dehydration of suspended solids in the water in biofloc technology, a module composed of 15 tanks of 200 liters were used, the tanks were inoculated with mature biofloc. For the aeration and movement of the water a blower of $0.5 \mathrm{hp}$ was used, the air was conducted to each tank 150 male tilapia with an average weight of 200 grams were used, from a pond with biofloc technology. The fishes were distributed randomly in the 15 tanks corresponding to the treatments the percentage of protein applied in the diet. Five repetitions were used for each treatment. The fishes of the treatments were fed with rations containing 24.28 and $32 \%$ protein, with a feeding rate of 4 . The environmental and water parameters were average water temperature $24.5, \mathrm{pH}$ 6.8 , sedimentable solids $50 \mathrm{ml} / \mathrm{I}$. To obtain the biofloc residue, a decanter was used to separate the solid part of the liquid by sedimentation, the residue obtained was dehydrated and analyzed. The objective of the present investigation was to know the nutritional and organoleptic properties of the biofloc meal, depending on the food consumed by the fish. The highest averages found were: protein $30.65 \pm 0.38$, fat $1.66 \pm 0.35$, dry matter $89.84 \pm 0.93$, ash $23.34 \pm 7.68$, fiber $17.76 \pm 7.68$; the smell was nice, It was brown color, and $50 \%$ of the flour was constituted by particles with a diameter of $170 \mu \mathrm{m}$.

Keywords: organic matter, biofloc, tilapia farming, technology

\section{Introducción}

Existen materias orgánicas de desecho con elevado contenido de proteína que puede llegar a ser el sustituto parcial de la harina de pescado y soya en las dietas de otros animales, una de estas alternativas es la harina procedente del residuo solido del biofloc del cultivo de peces. El sistema intensivo de producción con tecnología biofloc se muestra como una de las técnicas piscícolas más amigables con el medio ambiente, La tecnología biofloc; es una técnica de producción piscícola super intensiva, en la cual no se realizar recambios de agua, solo se repone la que se pierde por evaporación (Collazo y Arias, 2015).

En este sistema de producción se mantiene constante el flujo de aireación del estanque, el agua al estar en movimiento incrementa el oxígeno disuelto, además favorece la formación de la micro biota, que es el producto del medio acuático compuesto por microorganismos que consisten principalmente en bacterias, zooplancton, protozoos y microalgas, que se agregan a la materia orgánica del sistema; tales como las excretas, orina y residuos de alimento no consumido por los organismos cultivados. 


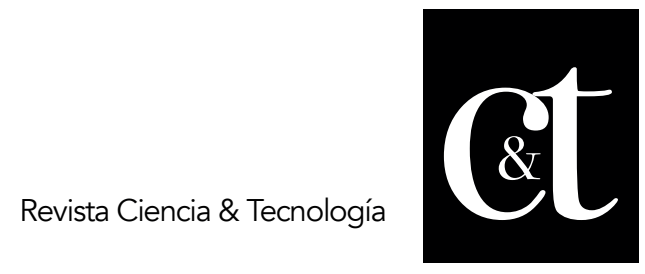

No. 20, 31 de octubre de 2018

ISSN impreso: 1390 - 6321

Al poner en suspensión los sedimentos del estanque permite que compuestos como el fósforo, carbono y nitrógeno principalmente, favorezcan el desarrollo de los microorganismos presentes en el agua y su biodiversidad, la cual se alojará en los agregados de materia orgánica o flóculos y dependerá de la microbiota que se encuentra en el agua (Ray et al., 2010). Los flóculos pueden proveer nutrientes importantes como las proteínas (Azim \& Little, 2008; Emerenciano et al., 2011), lípidos (Wasielesky et al., 2006; Emerenciano et al., 2012), aminoácidos (Ju et al., 2008) y ácidos grasos (Azim \& Little 2008; Ekasari \& Crab, 2010).

Por estas características, esta tecnología ha sido visualizada por investigaciones en el campo de la nutrición acuícola por ser una alternativa como fuente de alimento. Es fundamental que en este sistema se dé una adecuada aireación para conseguir que se desarrollen bacterias aerobias y que las partículas permanezcan suspendidas en la columna de agua (Avnimelech 2007; De Schryver et al., 2008; Newman 2011). además de contener entre 30 a $40 \%$ de materiales orgánicos como coloides, polímeros orgánicos y células muertas, los cuales pueden ser consumidos por otros organismos y reintegrados a las cadenas productivas (Avnimelech \& Kochba, 2009).

Estos flóculos microbianos combinan la extracción de los nutrientes presentes en el agua para la producción de biomasa, que puede ser usada en el cultivo de especies que sirvan de alimento (De Schryver et al., 2008). En un sistema biofloc se albergan microrganismos tales como (bacterias, zooplancton, protozoos y microalgas), que ayudan a la trasformación de la materia orgánica y son fuente de alimento para los peces, estos microorganismos se localizan en partículas denominadas flóculos (Emerenciano et al., 2013a). El biofloc (BF) es un agregado de microalgas, bacterias, protozoos, partículas de las heces, alimento no consumido, zooplancton y los nemátodos (Hargreaves, 2013).

El contenido de proteína del biofloc varía de 25 a 50\%; el contenido de grasa va de 0.5 a $15 \%$. Los bioflocs son buenas fuentes de vitamina y minerales, especialmente de fósforo; y podrían tener efectos probióticos (Aquahoy, 2013). Emerenciano et al. (2013b) y Hargreaves. (2013) destacan que los bioflocs proveen dos servicios críticos: el mantenimiento de la calidad del agua mediante la asimilación de los compuestos nitrogenados de los desechos provenientes de la alimentación; y provee una fuente de alimentos, con lo cual incrementa la factibilidad para reducir la tasa de conversión del alimento y disminuye los costos de la alimentación. La producción de biofloc en un estanque depende de la tasa de alimentación que se provea a los peces del estanque, considerando que en una conversión de alimento de 2 la aportación de nutrientes que se convertirán en biofloc equivale al 50 \% del alimento ofrecido.

La utilización del biofloc proveniente del estanque de la tecnología biofloc puede ser utilizada en la alimentación de otra especie animal, siendo así, con esta tecnología se evita que el residuo del cultivo de peces en la tecnología biofloc aporte a la 124

Rodríguez, Suin, González, Vivas, Díaz, Torres, Rodríguez. Análisis proximal de la harina de biofloc del cultivo de tilapia roja (oreochromis sp.) 


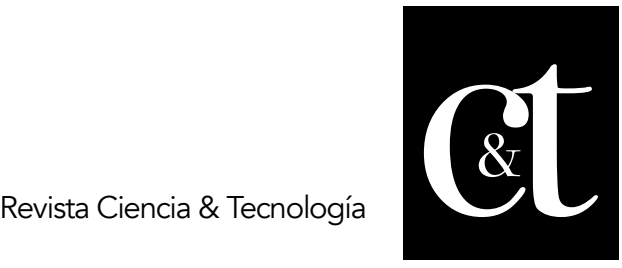

No. 20, 31 de octubre de 2018

ISSN impreso: 1390 - 6321

contaminación ambiental.

El presente trabajo tuvo como finalidad determinar el contenido nutricional de la harina de biofloc en el cultivo de tilapia roja alimentadas con diferentes porcentajes de proteína en la dieta.

\section{Desarrollo}

El presente trabajo se realizó en la Universidad Técnica Estatal de Quevedo. (UTEQ), ubicada en el cantón Quevedo, provincia de Los Ríos, Ecuador. El campo experimental está ubicado a $72 \mathrm{msnm}$, con una temperatura promedio anual de $25^{\circ} \mathrm{C}$ y 1000 milímetros de precipitación. Se utilizaron 15 tanques de 200 litros de capacidad, para la aireación del agua de los tanques se operó con un aireador de $0.5 \mathrm{hp}$, la distribución del aire a los tanques se la realizo por medio de manguera plástica de media pulgada, y para la difusión del aire al agua se utilizó $100 \mathrm{~cm}$ de manguera difusora de media pulgada, El agua de los tanques se inoculó con 4 galones de agua con biofloc proveniente del sistema de producción en biofloc del plantel piscícola de la UTEQ. Diariamente, a las 8,12 y 16 horas, se registró la temperatura externa e interna y los parámetros de calidad del agua en cada uno de los tanques: temperatura del agua, $\mathrm{pH}$, oxígeno disuelto (DO), ratio de sólidos disueltos (SDR).

El análisis de amoniaco y nitritos fue realizado semanalmente. Antes de ingresar los peces a los tanques, mantuvo en etapa de formación del biofloc hasta alcanzar $50 \mathrm{ml}$ /l de solidos suspendidos. En cada tanque se sembraron 10 tilapias rojas, (Oreochromis roja) machos de $200 \mathrm{~g}$ de peso, se procedió a alimentarlas tres veces al día con balanceado que contenía 24,28 y $32 \%$ de proteína, (correspondiente a los tratamientos 1,2 y 3), con una tasa de alimentación máxima de $4 \%$. Se utilizó un decantador para retirar el exceso de sólidos de los tanques cada siete días, los sólidos obtenidos son las muestras húmedas de biofloc. Las muestras fueron colocados en bandejas de plástico, identificadas con cada tratamiento, y se transportaron al laboratorio de bromatología de la Facultad de Ciencias Pecuarias de la Universidad Técnica Estatal de Quevedo, se deshidrató las muestras hasta alcanzar una humedad del $12 \%$, posteriormente se realizó el análisis proximal de: proteína, grasa, materia seca, minerales, grasa y fibra, además el análisis descriptivo sensorial de la harina de biofloc de los tratamientos, se identificó el olor, color y textura.

Se comprobó la adecuación de las condiciones experimentales del hábitat utilizado, a través de la respuesta de recolección de biofloc en cada estanque y la existencia de diferencias en los porcentajes de proteína, grasas, minerales, fibra presente en la harina de e biofloc en los tratamientos, mediante comparación de medias $(P<0,05)$.

Para evaluar los componentes de la harina de biofloc se realizó un análisis de varianza de una vía y ante la existencia de diferencias significativas se utilizó el test LSD de rangos múltiples $(P<0,05)$. Las variables de respuesta utilizadas fueron: porcentaje 
de proteína, grasas, minerales y fibra contenido en el biofloc deshidratado producido con tres dietas experimentales, para evaluar sus características de: olor, color y textura, además, determinar su composición proximal.

Para los análisis estadísticos se utilizó el programa SPSS versión 19.0 (IBM, 2010).

\section{Resultados}

Los resultados de proteína cruda indicaron que el mayor porcentaje de proteína lo obtuvo el tratamiento 3 , con un promedio de $30,65 \pm 0.38$, seguido por el tratamiento 2 con $27.20 \pm 0.23$ y en tercer lugar el tratamiento 1 con $19.15 \pm 1.06 \%$ de proteína, existiendo diferencia estadística significativa frente a los tratamientos 2 y 3 . El mayor porcentaje de grasa se encontró en el tratamiento 1 que obtuvo $1.66 \pm 0.35$, seguido del tratamiento 2 , con un porcentaje de grasa de $1.05 \pm 0.28$, posteriormente el tratamiento 3 que obtuvo $1.03 \pm 0.51$; aunque se observaron diferencias en contenido de grasa $(P<0.05)$ entre ambos tratamientos 2 y 3 frente al tratamiento 1.

El efecto del secado en estufa a 100 grados centígrados por 24 horas, determinó la ausencia significativa de humedad de las muestras que dieron los siguientes resultados de materia seca: el tratamiento 1 obtuvo $89.84 \pm 0.93$ de materia seca, seguido por el tratamiento 2 con $87.41 \pm 0.25$ y por último el tratamiento 3 con $87.39 \pm 0.31$, la humedad presente tuvo relación inversa con la cantidad de temperatura en la estufa aplicado a la muestra. Los porcentajes de humedad encontrados en los tratamientos 1,2 y 3.

El mayor contenido de energía lo obtuvo el tratamiento 3 con $3.90 \pm 0.21$, seguido por el tratamiento 1 con $3.55 \pm 0.31$, y el tratamiento 2 con $3.49 \pm 0.46$. El mayor porcentaje de ceniza se encontró en tratamiento 1 que se obtuvo, $23.34 \pm 7.68$, seguido del tratamiento 2 , con un porcentaje de ceniza de $20.05 \pm 1.67$, seguido del tratamiento 3 que se obtuvo $16.27 \pm 0.19$; se observaron diferencias en contenido de ceniza $(P<0.05)$ entre los tres tratamientos.

El mayor porcentaje de fibra se encontró en tratamiento 1 que se obtuvo, 17.76 \pm 7.68 , seguido del tratamiento 2 , con un porcentaje de fibra de $16.50 \pm 1.67$, seguido del tratamiento 3 que se obtuvo $15.10 \pm 0.19$; se observaron diferencias en contenido de ceniza $(P<0.05)$ entre los tres tratamientos.

En cuanto a color, se utilizó un colorímetro marca Lutron 1002, teniendo como resultado para el tratamiento 1 el código RBG los valores 208, 183, 1140, 8, respectivamente, para el tratamiento 2 los valores 174, 4147, 9114, 9, y para el tratamiento 3 los valores 163,3 138,6 105, 7, siendo la representación para estos valores el color pardo oscuro o marrón. En cuanto a olor, los resultado del olor fue determinado como agradable. Granulometría, el diámetro de las partículas analizadas 


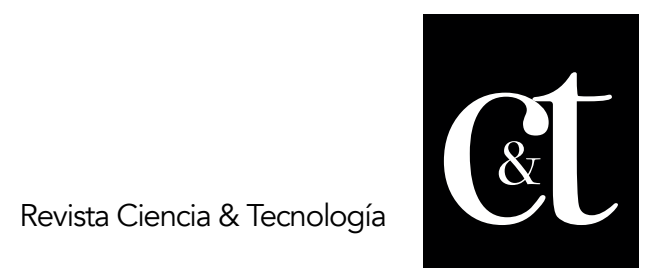

No. 20, 31 de octubre de 2018

ISSN impreso: 1390 - 6321

fue de 500,425 y $170 \mu \mathrm{m}$; el diámetro de $170 \mu \mathrm{m}$ dio como resultado 47.5, 45, y 53 $\%$ para los tratamientos 1,2 y 3 respectivamente.

\section{Conclusiones}

Los porcentajes de proteína encontrados están dentro del rango de proteína indicado por Hargreaves (2013), que reporta que el contenido de proteína en la harina de biofloc varía de 25 a $50 \%$, siendo los más común entre 30 y $45 \%$.; por otra parte, el mismo autor indica que la calidad nutricional del biofloc para los animales cultivados es bueno, pero variable.

Por su parte, Monroy-Dosta et al. (2013) en base a los resultados de su investigación en tilapia, confirmaron que los bioflocs contribuyen significativamente como fuente de alimento natural in situ, e incluye a comunidades microbianas heterotróficas del género Sphingomonas, Pseudomonas, Bacillus, Nitrospira, Nitrobacter y levadura Rhodotorula sp.

El porcentaje de grasa concuerda con lo reportado por Hargreaves (2013) quien indica que el contenido de grasas varía de 0.5 a $15 \%$, el más común se encuentra en el rango de 1 y $5 \%$. El mayor porcentaje del tamaño de las partículas estuvo en las $170 \mu \mathrm{m}$ que puede ser utilizada en la alimentación de pollos, considerando que Moran (1982) manifiesta que las partículas finas utilizadas en alimento para pollos aumentan la velocidad de tránsito en el intestino.

\section{Referencias bibliográficas}

Aquahoy (2013). Los sistemas de biofloc para la acuicultura. [Online]. Recuperado de:http://www.panoramaacuicola.com/noticias/2013/04/29/los_sistemas_de_b iofloc_para_la_acuicultura.html.

Avnimelech Y. (2007). Feeding with microbial flocs by tilapia in minimal discharge bio-flocs technology ponds. Aquaculture 264; 140-147.

Avnimelech Y. \& Kochba, M. (2009). Evaluation of nitrogen uptake and excretion by tilapia in bio floc tanks, using N-15 tracing. Aquaculture, v. 287, n. 1-2, p. 163$168,2009$.

Azim, M.E. \& Little, D. (2008). The biofloc technology (BFT) in indoor tanks: Water quality, biofloc composition, and growth and welfare of Nile tilapia (Oreochromis niloticus). Aquaculture 283(1-4):29-35. doi:10.1016/j.aquaculture.2008.06.036.

Collazo, L.F. y Arias, J. (2015) Fundamentos de la tecnología biofloc (BFT): Una alternativa para la piscicultura en Colombia. Orinoquia 19(1):77.

De Schryver, P, Crab, R, Defoirdt, T, Boon, N, \& Verstraete, W. (2008), The basics of 127 


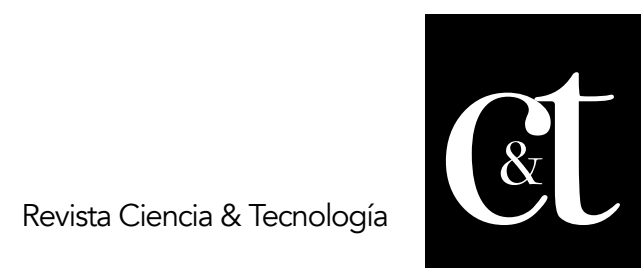

No. 20, 31 de octubre de 2018

ISSN impreso: 1390 - 6321

biofloc technology: the added value of aquaculture. Aquaculture 277: 125-137.

Ekasari, J. \& Crab, R. (2010). Primary Nutritional Content of Bio-Flocs Cultured with Different Organic Carbon Sources and Salinity. HAYATI Journal of Biosciences 17(3): 125-130.

Emerenciano, M., Ballester, E., Cavalli, R., \& Wasielesky, W. (2011). Effect of biofloc technology (BFT) on the early postlarval stage of pink shrimp Farfantepenaeus paulensis: growth performance, floc composition and salinity stress tolerance. Aquaculture International 19: 891-901.

Emerenciano, M., Ballester, E., Cavalli, R., \& Wasielesky, W. (2012). Biofloc technology application as a food source in a limited water exchange nursery system for pink shrimp Farfantepenaeus brasiliensis (Latreille, 1817). Aquaculture Research. 43, 447-457.

Emerenciano, M., Cuzon, G., Arévalo, M., \& Gaxiola, G. (2013a). Biofloc technology in intensive broodstock farming of the pink shrimp Farfantepenaeus duorarum: spawning performance, biochemical composition and fatty acid profile of eggs. Aquaculture Research. January. doi: 10.1111/are.12117

Emerenciano, M., Gaxiola, G., \& Cuzon, G. (2013b). Biofloc Technology (BFT): A Review for Aquaculture Application and Animal Food Industry, Biomass Now Cultivation and Utilization, Dr. Miodrag Darko Matovic (Ed.). DOI: 10.5772/53901. Recuperado de: https://www.intechopen.com/books/biomassnow-cultivation-and utilization/biofloc-technology-bft-a-review-for-aquacultureapplication-and- animal-food-industry.

Hargreaves, J. (2013). Biofloc Production Systems for aquaculture. United States: Southern Regional Aquaculture Center

Ju, Z., Forster, L., Conquest, W., Dominy, W., Kuo, W., \& Horgen, F. (2008). Determination of the microbial community structures of shrimp floc cultures by biomarkers and analysis of floc amino acid profiles. Aquaculture Research, 39: 118-133.

Monroy-Dosta, M., De Lara-Andrade R., Castro-Mejía, J., Castro-Mejía G. y Emerenciano, M. (2013). Composición y abundancia de comunidades microbianas asociadas al biofloc en un cultivo de tilapia. Revista de Biología Marina y Oceanografía. 48(3): 511-520.

Moran, E. (1982). Comparative nutrition of fowl and swine: the gastrointestinal systems. University of Guelph, Guelph, Ontario, Canadá.

128

Rodríguez, Suin, González, Vivas, Díaz, Torres, Rodríguez. Análisis proximal de la harina de biofloc del cultivo de tilapia roja (oreochromis sp.) 


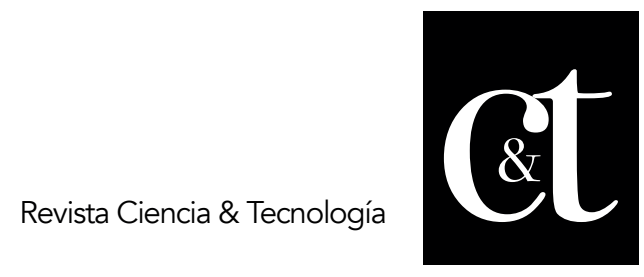

No. 20, 31 de octubre de 2018

ISSN impreso: 1390 - 6321

Ray, A., Seaborn, G., Leffler, J., Wilde, S., Lawson, A., \& Browdy, C. (2010). Characterization of the microbial communities in minimal exchange, intensive aquaculture systems and the effects of suspended solids management. Aquaculture. Vol. 310, 130-138.

Wasielesky, W, Atwood, H., Stokes, A. \& Browdy, C. (2006). Effect of natural production in brown water superintensive culture system for white shrimp Litopenaeus vannamei. Aquaculture 258: 396-403. 